\title{
BPS Alice strings
}

\section{Chandrasekhar Chatterjee and Muneto Nitta}

Department of Physics, and Research and Education Center for Natural Sciences, Keio University, Hiyoshi 4-1-1, Yokohama, Kanagawa 223-8521, Japan

E-mail: chandra@phys-h.keio.ac.jp, nitta@phys-h.keio.ac.jp

ABSTRACT: When a charged particle encircles around an Alice string, it changes the sign of the electric charge. In this paper we find a BPS-saturated Alice string in $\mathrm{U}(1) \times \mathrm{SO}(3)$ gauge theory with charged complex scalar fields belonging to the vector representation. After performing BPS completion we solve the BPS equations numerically. We further embed the Alice string into an $\mathcal{N}=1$ supersymmetric gauge theory to show that it is half BPS.

Keywords: Solitons Monopoles and Instantons, Supersymmetric Gauge Theory, Gauge Symmetry, Spontaneous Symmetry Breaking

ARXIV EPRINT: 1703.08971 


\section{Contents}

1 Introduction 1

2 The model for BPS Alice strings and the BPS equations 3

3 A BPS Alice string solution 4

$\begin{array}{lll}4 & \text { Symmetry structure of the Alice string } & 6\end{array}$

5 The SUSY model and 1/2 BPS Alice strings 4

$\begin{array}{lll}6 & \text { Summary and discussion } & 10\end{array}$

\section{Introduction}

An Alice string was discovered theoretically almost four decades ago by Albert S. Schwarz [1]. This string has a non-trivial topological property that charged particles flip the sign of the electric charge after encircling the Alice string once, and consequently the charge is not globally defined. The theory admitting an Alice string is sometime called as "Alice electrodynamics", which is a U(1) gauge theory that includes the charge conjugation as a local symmetry, as was discussed first time even before by Joe Kiskis [2]. A typical Alice string was found in an $\mathrm{SO}(3)$ gauge theory with scalar fields in spin-2 (traceless symmetric tensor) representation of $\mathrm{SO}(3)$. The $\mathrm{SO}(3)$ gauge group is spontaneously broken to $\mathrm{O}(2)$, giving rise to the vacuum manifold or order parameter space $G / H=\mathrm{SO}(3) / \mathrm{O}(2) \simeq \mathbb{R} P^{2}$. The non-trivial homotopy group $\pi_{1}\left(\mathbb{R} P^{2}\right) \simeq \mathbb{Z}_{2}$ admits an Alice string. Then, the unbroken generator, which we identify as the electromagnetic U(1) generator, changes its sign when encircling the Alice string once. A possible application of Alice strings in cosmology was proposed in ref. [3].

The Alice string is an example of a wide class of systems of spontaneously broken symmetry where the unbroken symmetry is either a discrete group or a continuous group that contains components which are not connected to the identity. In the case of the Alice string, the unbroken gauge group is $\mathrm{O}(2)$ which is a continuous group with more than one connected components. In general, embedding of the unbroken group becomes space dependent inside the full symmetry group in the presence of vortices. The presence of discrete symmetry or disconnected components in the unbroken gauge group often makes some of the generators undefined globally [4-6]. In the case of the Alice string, the generator of unbroken U(1) group changes the sign after encircling once around the vortex. This shows a very interesting exotic phenomenon called the creation of "Cheshire charge", a charge which is lost after charged particles encircle the string once [7-9]. This 
allows all kinds of interesting global effects, such as the generalized Aharonov-Bohm effect, anyonic exchange statistics etc [10]. Not only electric Cheshire charge, the Alice string also exchanges magnetic charges from magnetic particles, creating the Cheshire magnetic charges [9]. Interesting thing is that a ring of the Alice string can be turned into a magnetic monopole at large distances once the U(1) modulus is twisted along the ring [11-14]. This follows from the fact that in case of the Alice string, the spontaneous symmetry breaking gives non-trivial second homotopy group, $\pi_{2}(G / H) \neq 0$.

On the other hand, Bogomol'nyi-Prasad-Sommerfield (BPS) states are the most stable states among configurations with a fixed topological charge since they saturate Bogomol'nyi bound of the energy $[15,16]$. In this case, the total energy is proportional to the topological charge, namely the number of solitons. Abrikosov-Nielsen-Olesen (ANO) vortices (flux tubes) $[17,18]$ become BPS at the critical coupling between type-I and type-II superconductors. Such BPS vortices preserve a half supersymmetry (SUSY) when embedded into SUSY gauge theories. They belong to so-called short SUSY multiplets and consequently are stable against quantum corrections [19]. Therefore they play crucial roles to determine non-perturbative effects of the theories. BPS non-Abelian vortices are also present in certain SUSY non-Abelian gauge theories [20-28]. On the other hand, the symmetry breaking which creates conventional Alice strings [1] gives the fundamental group $\pi_{1}(G / H) \simeq \mathbb{Z}_{2}$, implying that an Alice string can annihilate itself when two of them collide, contradict to a BPS property that energy is proportional to the topological charge. Therefore, Alice strings are naturally non-BPS. ${ }^{1}$

Another type of Alice strings is known in Bose-Einstein condensates (BEC) [32], see also $[33,34]$. More presicely, the theory is a spinor BEC with scalar fields belonging to vector (spin-1) representation of $\mathrm{SO}(3)$, for which a group $\mathrm{U}(1) \times \mathrm{SO}(3)$ is spontaneously broken to $\mathrm{O}(2)$ giving an order parameter space $G / H=[\mathrm{U}(1) \times \mathrm{SO}(3)] / \mathrm{O}(2) \simeq\left[S^{1} \times S^{2}\right] / \mathbb{Z}_{2}$ with a non-trivial homotopy group $\pi_{1}(G / H) \simeq \mathbb{Z}$. This admits a global analog of Alice strings but one does not have a charged particle since all symmetries $G$ are global symmetries.

In this paper, we find that a BPS Alice string exists in a $\mathrm{U}(1) \times \mathrm{SO}(3)$ gauge theory coupled with charged complex scalar fields in the vector representation. This is achieved simply by gauging the symmetry $G=\mathrm{U}(1) \times \mathrm{SO}(3)$ in the corresponding spin- 1 BEC model [32]. We numerically construct an axially symmetric Alice string configuration, and find that equations for profile functions for it coincide with those for a non-Abelian vortex [21] in $\mathrm{U}(N)$ gauge theory coupled with $N$ complex Higgs fields in the fundamental representation. We further embed our bosonic model into an $\mathcal{N}=1$ SUSY gauge theory and show that the BPS Alice string is a $1 / 2$ BPS state, preserving a half SUSY.

This paper is organized as follows. In section 2 we introduce a simple model of $U(1) \times$ $\mathrm{SO}(3)$ gauge theory with a complex scalar field in a vector representation. We perform the Bogomol'nyi completion of the energy functional and write down the BPS equations. In section 3 we introduce an ansatz and boundary condition for a single Alice string. We

\footnotetext{
${ }^{1} \mathrm{~A}$ BPS Alice string in $\mathcal{N}=4$ SUSY gauge theory was discussed without an explicit construction in string theory $[29,30]$. There is also an attempt in ref. [31] to obtain a BPS Alice string of the conventional type but no Bogomol'nyi completion could be achieved, where the system is forced to become of first order by assuming some constraints by hand in a particular gauge.
} 
expand BPS equations in the profile functions and solve the equations numerically. In section 4 we discuss a symmetry structure of our Alice string. Section 5 is devoted to a summary and discussion.

\section{The model for BPS Alice strings and the BPS equations}

We consider an $\mathrm{SO}(3) \times \mathrm{U}(1)$ gauge theory coupled with charged complex scalar fields in the vector representation, equivalently an $\mathrm{SU}(2) \times \mathrm{U}(1)$ gauge theory coupled with one charged complex scalar field in the adjoint representation. Here we use the latter expression for usefulness. We denote $\mathrm{SU}(2)$ and $\mathrm{U}(1)$ gauge fields by $A_{\mu}=A_{\mu}^{a} \tau^{a}$ and $a_{\mu}$, respectively, and a charged complex scalar field in the adjoint representation by $\Phi=\Phi^{a} \tau^{a}$, where $\tau^{a}=\frac{1}{2} \sigma^{a}$ and $\sigma^{a}(a=1,2,3)$ are the Pauli matrices. The action is given by

$$
I=\int d^{4} x\left[-\frac{1}{2} \operatorname{Tr} F_{\mu \nu} F^{\mu \nu}-\frac{1}{4} f_{\mu \nu} f^{\mu \nu}+\operatorname{Tr}\left|D_{\mu} \Phi\right|^{2}-\frac{\lambda_{g}}{4} \operatorname{Tr}\left[\Phi, \Phi^{\dagger}\right]^{2}-\frac{\lambda_{e}}{2}\left(\operatorname{Tr} \Phi \Phi^{\dagger}-2 \xi^{2}\right)^{2}\right] .
$$

The covariant derivatives and field strengths are defined by $D_{\mu} \Phi=\partial_{\mu} \Phi-i e a_{\mu} \Phi-i g\left[A_{\mu}, \Phi\right]$, and $F_{\mu \nu}=\partial_{\mu} A_{\nu}-\partial_{\nu} A_{\mu}-i g\left[A_{\mu}, A_{\nu}\right], f_{\mu \nu}=\partial_{\mu} a_{\nu}-\partial_{\nu} a_{\mu}$, with the gauge couplings $g$ and $e$ for $\mathrm{SU}(2)$ and $\mathrm{U}(1)$ gauge fields, respectively. Since the center of $\mathrm{SU}(2)$ does not acts on the adjoint field, the gauge symmetry is actually $G=\mathrm{U}(1)_{b} \times \frac{\mathrm{SU}(2)}{\mathbb{Z}_{2}} \simeq \mathrm{U}(1)_{b} \times \mathrm{SO}(3)$.

In the vacuum, the scalar field takes the non-zero vacuum expectation value as

$$
\langle\Phi\rangle=2 \xi \tau^{1}
$$

which keeps the $\mathrm{U}(1)_{1}$ symmetry generated by $\tau^{1}$ unbroken and consequently $A_{\mu}^{1}$ remains massless, while other gauge fields become massive. The breaking pattern of the gauge symmetry is

$$
G=\mathrm{U}(1)_{b} \times \frac{\mathrm{SU}(2)}{\mathbb{Z}_{2}} \simeq \mathrm{U}(1)_{b} \times \mathrm{SO}(3) \longrightarrow H \simeq \mathbb{Z}_{2} \ltimes \mathrm{U}(1)_{1} \simeq \mathrm{O}(2)
$$

where $\ltimes$ denotes a semi-direct product. The unbroken $\mathbb{Z}_{2}$ is given by a simultaneous action of a $\pi$ rotation around either $\tau^{3}, \tau^{2}$ or their linear combination and a $\pi$ rotation in $\mathrm{U}(1)_{b}$. Since the $\mathbb{Z}_{2}$ action does not commute with $\mathrm{U}(1)_{1}$, there is the semi-direct product $\ltimes$ between them. The vacuum manifold is obtained as

$$
\frac{G}{H}=\frac{\mathrm{U}(1)_{b} \times \mathrm{SO}(3)}{\mathrm{O}(2)} \simeq \frac{S^{1} \times S^{2}}{\mathbb{Z}_{2}}
$$

This allows a non-trivial fundamental group

$$
\pi_{1}(G / H) \simeq \mathbb{Z}
$$

indicating the existence of stable vortices. One can observe that compared with the vacuum manifold $S^{2} / \mathbb{Z}_{2} \simeq \mathbb{R} P^{2}$ of the conventional Alice string, there is the U(1) factor in eq. (2.4). We will see that this difference is essential for the BPS property. 
The static energy is expressed as

$$
\mathcal{H}=\int d^{3} x\left[\frac{1}{2} \operatorname{Tr} F_{i j}^{2}+\frac{1}{4} f_{i j}^{2}+\operatorname{Tr}\left|D_{i} \Phi\right|^{2}+\frac{\lambda_{g}}{4} \operatorname{Tr}\left[\Phi, \Phi^{\dagger}\right]^{2}+\frac{\lambda_{e}}{2}\left(\operatorname{Tr} \Phi \Phi^{\dagger}-2 \xi^{2}\right)^{2}\right] .
$$

Here, we consider the critical couplings,

$$
\lambda_{e}=e^{2}, \quad \lambda_{g}=g^{2}
$$

at which we can consider the Bogomol'nyi's arguments. By performing the Bogomol'nyi completion, the tension (energy per the unit length) of a vortex along the $x_{3}$ coordinate can be written as

$$
\begin{aligned}
\mathcal{T} & =\int d^{2} x\left[\operatorname{Tr}\left[F_{12} \pm \frac{g}{2}\left[\Phi, \Phi^{\dagger}\right]\right]^{2}+\operatorname{Tr}\left|\mathcal{D}_{ \pm} \Phi\right|^{2}+\frac{1}{2}\left[f_{12} \pm e\left(\operatorname{Tr} \Phi \Phi^{\dagger}-2 \xi^{2}\right)\right]^{2} \pm 2 e f_{12} \xi^{2}\right] \\
& \geq 2 e \xi^{2}\left|\int d^{2} x f_{12}\right|
\end{aligned}
$$

where $\mathcal{D}_{ \pm} \equiv \frac{D_{1} \pm i D_{2}}{2}$ are used together with the complex coordinate $z \equiv x_{1}+i x_{2}$, and all fields are taken to be independent of $x_{3}$ coordinate. The BPS condition is satisfied when the inequality is saturated, in which case the tension is simply given by

$$
\mathcal{T}_{\mathrm{BPS}}=2 e \xi^{2}\left|\int d^{2} x f_{12}\right|
$$

At this saturation point we can write first order BPS equations

$$
\begin{aligned}
f_{12} \pm e\left(\operatorname{Tr} \Phi \Phi^{\dagger}-2 \xi^{2}\right) & =0, \\
F_{12} \pm \frac{g}{2}\left[\Phi, \Phi^{\dagger}\right] & =0, \\
\mathcal{D}_{ \pm} \Phi=\left(\mathcal{D}_{ \pm} \Phi\right)^{\dagger} & =0 .
\end{aligned}
$$

All configurations that satisfy the above BPS equation also satisfy the full second order equations of motion as usual.

\section{A BPS Alice string solution}

In this section, we construct a cylindrically symmetric single vortex solution. To solve the BPS equations, we consider the ansatz

$$
\begin{aligned}
\Phi(r, \varphi) & =\xi\left(\begin{array}{cc}
0 & f_{1}(r) e^{i \varphi} \\
f_{2}(r) & 0
\end{array}\right), \\
a_{i}(r, \varphi) & =-\frac{1}{2 e} \frac{\epsilon_{i j x_{j}}}{r^{2}} a(r), \quad A_{i}(r, \varphi)=-\frac{1}{4 g} \frac{\epsilon_{i j x_{j}}}{r^{2}} \sigma^{3} A(r) .
\end{aligned}
$$

where $\{r, \varphi\}$ are radius and azimuthal angle, respectively. Here, $f_{1}(r), f_{2}(r), A(r)$ and $a(r)$ are four profile functions depending only on the radial coordinate. Then, the first order 
equations take the form

$$
\begin{aligned}
\frac{1}{r} \partial_{r} a(r)+2 e^{2} \xi^{2}\left[f_{1}(r)^{2}+f_{2}(r)^{2}-2\right] & =0, \\
\frac{1}{r} \partial_{r} A(r)+2 g^{2} \xi^{2}\left[f_{1}(r)^{2}-f_{2}(r)^{2}\right] & =0, \\
\partial_{r} f_{1}(r)-\frac{1}{r}\left(1-\frac{a(r)+A(r)}{2}\right) f_{1}(r) & =0, \\
\partial_{r} f_{2}(r)+\frac{a(r)-A(r)}{2 r} f_{2}(r) & =0 .
\end{aligned}
$$

We solve the above equations with the boundary conditions

$$
\begin{array}{ll}
f_{1}(0)=f_{2}^{\prime}(0)=0, & f_{1}(\infty)=f_{2}(\infty)=1, \\
A(0)=a(0)=0, & A(\infty)=a(\infty)=1 .
\end{array}
$$

To solve these equations let us define two new profile functions $\psi_{0}(r)$ and $\psi_{1}(r)$ as

$$
f_{1}(r)=r e^{-\frac{1}{2}\left(\psi_{0}(r)+\psi_{1}(r)\right)}, \quad f_{2}(r)=e^{\frac{1}{2}\left(\psi_{1}(r)-\psi_{0}(r)\right)} .
$$

These give

$$
A(r)=r \psi_{1}^{\prime}(r), \quad a(r)=r \psi_{0}^{\prime}(r) .
$$

Then, the above four equations are reduced to the following two equations,

$$
\begin{aligned}
\frac{1}{\rho} \partial_{\rho}\left[\rho \psi_{0}^{\prime}(\rho)\right]+l^{2}\left[e^{-\psi_{0}}\left(\rho^{2} e^{-\psi_{1}}+e^{\psi_{1}}\right)-2\right] & =0, \\
\frac{1}{\rho} \partial_{\rho}\left[\rho \psi_{1}^{\prime}(\rho)\right]+e^{-\psi_{0}}\left(e^{-\psi_{1}}-e^{\psi_{1}}\right) & =0,
\end{aligned}
$$

where we have defined $\rho^{2} \equiv 2 g^{2} \xi^{2} r^{2}$ and the ratio $l \equiv e / g$ between the $\mathrm{U}(1)$ and $\mathrm{SO}(3)$ gauge couplings. The boundary conditions for the new variables become

$$
\psi_{0}^{\prime}(0)=\psi_{1}^{\prime}(0), \quad \psi_{0}(R)=\psi_{1}(R)=\log R,
$$

where $R$ is the system size. We solved these equations numerically and all the profile functions are plotted in figure 1.

We may further rewrite the equations by defining

$$
\tilde{\psi}_{1}=\psi_{1}-\log \rho \quad \tilde{\psi}_{0}=\psi_{0}-\log \rho
$$

and these redefinitions transform the equations to the form

$$
\begin{aligned}
\frac{1}{\rho} \partial_{\rho}\left[\rho \tilde{\psi}_{0}^{\prime}(\rho)\right]+2 l^{2}\left[e^{-\tilde{\psi}_{0}} \cosh \tilde{\psi}_{1}-1\right] & =-2 \pi \delta^{2}(\rho), \\
\frac{1}{\rho} \partial_{\rho}\left[\rho \tilde{\psi}_{1}^{\prime}(\rho)\right]-2 e^{-\tilde{\psi}_{0}} \sinh \tilde{\psi}_{1} & =-2 \pi \delta^{2}(\rho) .
\end{aligned}
$$

For $l=1$ these two equations posses the solution $\psi_{1}(\rho)=\psi_{0}(\rho)$, and the above two equations are reduced to a single equation, which is known as the Taubes equation [35] for a single ANO vortex [18]. 


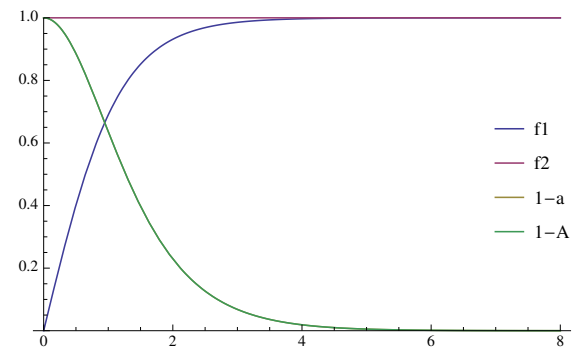

(a)

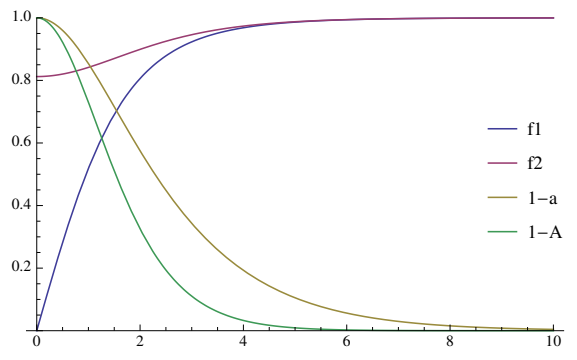

(b)

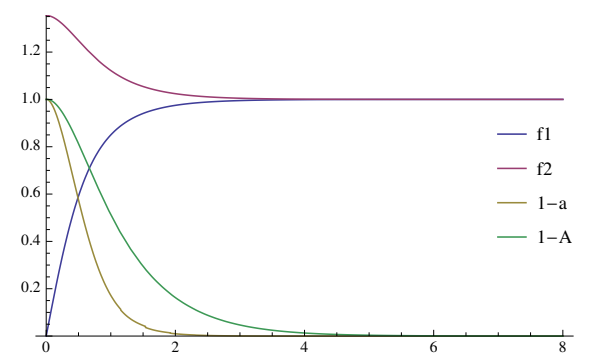

(c)

Figure 1. The profile functions $f_{1}(\rho), f_{2}(\rho), 1-a(\rho)$ and $1-A(\rho)$ as functions of the distance $\rho$ from the vortex center for a single Alice string vortex. We plot them for different values of the ratio $l=\frac{e}{g}$ between the $\mathrm{U}(1)$ and $\mathrm{SO}(3)$ gauge couplings. (a) $l=1$, (b) $l=\frac{1}{2}$, (c) $l=5$.

Before closing this section, it may be interesting to note that eqs. (3.3)-(3.6) for profile functions are identical to those of an axially symmetric non-Abelian vortex in $\mathrm{U}(N)$ gauge theory coupled with $N$ flavors in the fundamental representations [21]. Although the physical properties are quite different between them, some mathematical properties such as the uniqueness and existence of the solutions are common.

\section{Symmetry structure of the Alice string}

In this section we shall analyze the symmetries and symmetry breaking of the system. To understand the symmetry breaking defined in eq. (2.3), let us analyze the large distance behavior of the order parameter of the scalar field. The order parameter has a non-trivial winding at large distances of the string and can be expressed as

$$
\Phi(R, \varphi) \sim \xi e^{i \frac{\varphi}{2}}\left(\begin{array}{cc}
0 & e^{i \frac{\varphi}{2}} \\
e^{-i \frac{\varphi}{2}} & 0
\end{array}\right)=\xi e^{i \frac{\varphi}{2}} e^{i \frac{\varphi}{4} \sigma^{3}} \sigma^{1} e^{-i \frac{\varphi}{4} \sigma^{3}}
$$

with the system size $R$. Now if we set the order parameter at $\varphi=0$ (along the $x_{1}$-axis) as

$$
\Phi(R, \varphi=0)=\xi \sigma^{1},
$$

the order parameter at any arbitrary $\varphi$ can be obtained by a holonomy action as

$$
\begin{aligned}
\Phi(R, \varphi) & =e^{i e \int \mathbf{a} \cdot \mathbf{d} \mathbf{l}} e^{i g \int \mathbf{A} \cdot \mathbf{d} \mathbf{l}} \Phi(R, 0) e^{-i g \int \mathbf{A} \cdot \mathbf{d} \mathbf{l}} \\
& =U_{0}(\varphi) U_{3}(\varphi) \Phi(\infty, 0) U_{3}^{-1}(\varphi)
\end{aligned}
$$




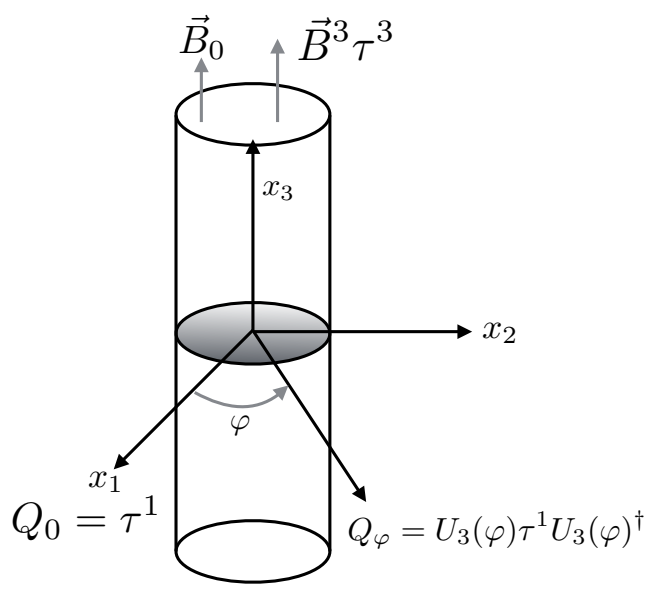

Figure 2. The Alice string is schematically shown. The Abelian and non-Abelian magnetic fields along the $x_{3}$ axis are denoted by $\vec{B}^{0}$ and $\vec{B}^{3}$, respectively. The change of the electric charge of the unbroken $\mathrm{U}(1)$ is displayed by denoting $Q_{0}$ at $\varphi=0$ and $Q_{\varphi}$ for arbitrary azimuthal angle outside the string.

where we have defined holonomies by

$$
U_{0}(\varphi)=e^{i e \int_{0}^{\varphi} \mathbf{a} \cdot \mathbf{d} \mathbf{l}}=e^{i \frac{\varphi}{2}}, \quad U_{3}(\varphi)=P e^{i g \int_{0}^{\varphi} \mathbf{A} \cdot \mathbf{d} \mathbf{l}}=e^{i \frac{\varphi}{4} \sigma^{3}} .
$$

These are obtained by the condition that the order parameter $\Phi$ is covariantly constant at large distances $\left(\mathcal{D}_{i} \Phi \rightarrow 0\right.$ as $\left.R \rightarrow \infty\right)$.

Now it can be easily noticed that the generator of unbroken group changes as we encircle the string by an angle $\varphi$. The generator of the unbroken $\mathrm{U}(1)$ at $\varphi=0$ is defined as $Q_{0}=\tau^{1}$. Then the $\mathrm{U}(1)$ generator at the angle $\varphi$ can be obtained by an action of the holonomy $U_{3}(\varphi)$ on $Q_{0}$ as

$$
Q_{\varphi}=U_{3}(\varphi) Q_{0} U_{3}(\varphi)^{-1}, \quad U_{3}(\varphi)=e^{\frac{i \varphi}{4} \sigma^{3}} .
$$

We then find that the generator changes its sign after the completion of one full circle around the vortex as

$$
Q_{2 \pi}=-Q_{0}
$$

This makes the non-existence of electric charge globally, as the case of conventional Alice strings.

The action of holonomy in eq. (4.4) can be written in the $\mathrm{SO}(3)$ spin one representation as

$$
J_{3}=\left(\begin{array}{ccc}
1 & 0 & 0 \\
0 & 0 & 0 \\
0 & 0 & -1
\end{array}\right), \quad U_{3}(\varphi)=e^{\frac{i \varphi}{2} J_{3}}, \quad U_{3}(0)=\mathbf{1}, \quad U_{3}(2 \pi)=\operatorname{diag} .(-1,1,-1) .
$$

This shows that

$$
U_{3}(2 \pi) \in \mathbb{Z}_{2} \subset \mathrm{O}(2)
$$

implying the Alice property more clearly. 
Before closing this section, let us make a remark. The above Alice phenomenon is an example of "obstruction" [36-39]. In general, a covariantly constant embedding of the unbroken symmetry group $H$ (the little group of $\Phi(\varphi)$ ) inside the original symmetry group $G$ depends on $\varphi$. If $H$ is a non-Abelian discrete group or a continuous group containing discrete elements as a semi-direct product, some generators become multivalued. Such a system in which a gauge group contains disconnected elements was discussed in ref. [2] in the context of $\mathrm{O}(2)$ gauge theory where charge conjugation symmetry $\left(\mathbb{Z}_{2}\right)$ was introduced as a local symmetry along with the $\mathrm{SO}(2)$ gauge theory in a non-simply connected space.

\section{The SUSY model and 1/2 BPS Alice strings}

In this section, we embed the bosonic action in the last sections into an $\mathcal{N}=1$ SUSY gauge theory in $3+1$ dimensions (or equivalently $\mathcal{N}=2$ SUSY theory in $2+1$ dimensions). Then, we show that BPS equations can be obtained by a half SUSY preserving condition.

Let us introduce chiral superfields $\Psi_{ \pm}(x, \theta, \bar{\theta})$ transforming under the adjoint representation of the $\mathrm{SU}(2)$ group with the $\mathrm{U}(1)$ charge \pm 1 , and the vector superfields $V_{0}(x, \theta, \bar{\theta})$ and $V(x, \theta, \bar{\theta})=V^{a}(x, \theta, \bar{\theta}) \tau^{a}$ for the $\mathrm{U}(1)$ and $\mathrm{SU}(2)$ gauge groups, respectively. Then, the $\mathcal{N}=1$ SUSY action that we consider is given in terms of the above superfields by

$$
\begin{aligned}
\mathcal{I}= & -\frac{1}{4} \Re \int d^{4} x d^{2} \theta\left(2 \operatorname{Tr} W^{\alpha} W_{\alpha}+W_{0}^{\alpha} W_{\alpha}^{0}\right) \\
& +\frac{1}{4} \int d^{4} x d^{2} \theta d^{2} \bar{\theta} \operatorname{Tr}\left(\Psi_{+}^{\dagger} e^{-2 e V_{0}-2 g V} \Psi_{+}+\Psi_{-}^{\dagger} e^{2 e V_{0}-2 g V} \Psi_{-}-\xi^{2} V_{0}\right)
\end{aligned}
$$

where $\xi^{2}$ is a constant called the Fayet-Iliopoulos parameter. ${ }^{2}$

The above action can be expanded in terms of component fields as

$$
\begin{aligned}
\mathcal{L}= & -\frac{1}{4 e^{2}} f_{\mu \nu}^{2}-\frac{1}{4 g^{2}} F_{\mu \nu}^{a} F_{\mu \nu}^{a}+i \lambda_{0}^{\dagger} \bar{\sigma}^{\mu} \partial_{\mu} \lambda_{0}+i \lambda^{\dagger a} \bar{\sigma}^{\mu}\left(\mathcal{D}_{\mu} \lambda\right)^{a} \\
& +\sum_{i= \pm} \operatorname{Tr}\left[\left(\mathcal{D}_{\mu} \Phi_{i}\right)_{a}^{\dagger} \mathcal{D}_{\mu} \Phi_{a}^{i}+\bar{\psi}_{i}^{a} \bar{\sigma}^{\mu}\left(\mathcal{D} \psi_{i}\right)^{a}+i \sqrt{2} \Phi_{i}^{\dagger}\left\{\lambda, \psi_{i}\right\}-i \sqrt{2}\left\{\bar{\psi}_{i}, \bar{\lambda}\right\} \Phi_{i}\right. \\
& \left.\quad+D\left[\Phi_{i}, \Phi_{i}^{\dagger}\right]+F_{i}^{\dagger} F_{i}\right] \\
& +\frac{1}{2} D_{a}^{2}+\frac{1}{2} D_{0}^{2}+D_{0} \operatorname{Tr}\left[\Phi_{+}^{\dagger} \Phi_{+}-\Phi_{-}^{\dagger} \Phi_{-}-\xi^{2}\right]+\operatorname{Tr}\left[i \sqrt{2} \Phi_{+}^{\dagger} \psi_{+} \lambda_{0}-i \sqrt{2} \bar{\psi}_{+} \bar{\lambda}_{0} \Phi_{+}\right] \\
& -\operatorname{Tr}\left[i \sqrt{2} \Phi_{-}^{\dagger} \psi_{-} \lambda_{0}-i \sqrt{2} \bar{\psi}_{-} \bar{\lambda}_{0} \Phi_{-}\right] .
\end{aligned}
$$

The auxiliary fields $D, D^{0}$ and $F^{ \pm}$can be solved as

$$
D_{0}=2 \xi^{2}-\operatorname{Tr}\left(\Phi_{+}^{\dagger} \Phi_{+}-\Phi_{-}^{\dagger} \Phi_{-}\right), \quad D=-\frac{1}{2} \sum_{i= \pm}\left[\Phi_{i}, \Phi_{i}^{\dagger}\right], \quad F^{ \pm}=0 .
$$

\footnotetext{
${ }^{2}$ The cosmic strings in the same theory were also discussed before in ref. [40]. However explicit Bogomol'nyi completion and BPS vortex solutions were not discussed there, and they seem to be unaware of the fact that these are Alice strings even though in the same theory.
} 
If we set $\Phi_{-}=0$ and $\Phi_{+}=\Phi$, we then can recover the action in eq. (2.1) in bosonic fields, for which the potential term is

$$
V(\Phi)=\frac{g^{2}}{4} \operatorname{Tr}\left[\Phi, \Phi^{\dagger}\right]^{2}+\frac{e^{2}}{2}\left(\operatorname{Tr} \Phi \Phi^{\dagger}-2 \xi^{2}\right)^{2} .
$$

$\Phi_{-}$parametrizes a flat direction of the theory. The effect of a flat direction on BPS vortices of the ANO type was discussed in refs. [41, 42].

Now we show that the BPS equations can be obtained by imposing SUSY transformation of the fermions to be zero. The SUSY transformations of the fermions can be written as

$$
\begin{aligned}
\delta_{\epsilon} \psi_{ \pm} & =i \sqrt{2} \sigma^{\mu} \bar{\epsilon} \mathcal{D}_{\mu} \Phi_{ \pm}+\sqrt{2} \epsilon F_{ \pm} \\
\delta_{\epsilon} \lambda^{a} & =\sigma^{\mu \nu} \epsilon F_{\mu \nu}^{a}+i \epsilon D^{a} \\
\delta_{\epsilon} \lambda^{0} & =i \epsilon D_{0}+\sigma^{\mu \nu} \epsilon f_{\mu \nu} .
\end{aligned}
$$

Here we have imposed the condition

$$
\left\langle\Psi_{-}\right\rangle=0
$$

By using above condition (5.8) and eq. (5.3), we can recover the BPS equations by setting the SUSY transformation of the fermions to be zero:

$$
\begin{aligned}
\delta_{\epsilon} \psi_{+} & =i \sqrt{2} \sigma^{\mu} \bar{\epsilon} \mathcal{D}_{\mu} \Phi_{+}+\sqrt{2} \epsilon F_{+}=0 \\
& \Rightarrow\left\{\left(D_{1}+i D_{2}\right) \Phi_{+}\right\} \bar{\epsilon}^{\dot{1}}=\left\{\left(D_{1}-i D_{2}\right) \Phi_{+}\right\} \bar{\epsilon}^{\dot{2}}=0 \\
\delta_{\epsilon} \lambda^{a} & =\sigma^{\mu \nu} \epsilon F_{\mu \nu}^{a}+i \epsilon D^{a}=0 \\
& \Rightarrow\left(F_{12}+\frac{1}{2}\left[\Phi_{+}, \Phi_{+}^{\dagger}\right]\right) \epsilon_{1}=\left(F_{12}-\frac{1}{2}\left[\Phi_{+}, \Phi_{+}^{\dagger}\right]\right) \epsilon_{2}=0,
\end{aligned}
$$

and

$$
\begin{aligned}
\delta_{\epsilon} \lambda^{0} & =i \epsilon D_{0}+\sigma^{\mu \nu} \epsilon f_{\mu \nu}=0 \\
& \Rightarrow\left\{f_{12}+e\left(\operatorname{Tr} \Phi_{+} \Phi_{+}^{\dagger}-2 \xi^{2}\right)\right\} \epsilon_{1}=\left\{f_{12}-e\left(\operatorname{Tr} \Phi_{+} \Phi_{+}^{\dagger}-2 \xi^{2}\right)\right\} \epsilon_{2}=0 .
\end{aligned}
$$

We then find that the condition

$$
\epsilon_{2}=\epsilon^{\dot{2}}=0
$$

gives the BPS equations (2.10)-(2.12) with the upper sign, while the condition

$$
\epsilon_{1}=\epsilon^{i}=0
$$

gives the same equations with the lower sign. We thus have shown that a BPS Alice string preserves a half of SUSY and is a 1/2 BPS state. 


\section{Summary and discussion}

In this paper, we have presented a BPS construction of an Alice string in a non-Abelian gauge theory. To this end, we have considered a simple model of $\mathrm{U}(1) \times \mathrm{SO}(3)$ gauge theory with charged complex scalar fields in the vector representation. To find a vortex solution, we first have written down the BPS equation by the Bogomol'nyi completion of the energy functional. We then have written our ansatz for the scalar and gauge fields for an axially symmetric single vortex. There are total four profile functions but BPS equations reduce them into two $\left(f_{1}(\rho), f_{2}(\rho)\right)$. We have solved the profile functions numerically to construct solutions. We have found that equations for profile functions are the same with those of an axially symmetric non-Abelian vortex in $\mathrm{U}(N)$ gauge theory coupled with $N$ fundamental representation. We then embed our Alice string in an $\mathcal{N}=1$ SUSY action by introducing two chiral superfields along with vector superfields corresponding to $\mathrm{U}(1)$ and $\mathrm{SU}(2)$ gauge theories with the Fayet-Iliopoulos term. The vortex is found to be 1/2-BPS saturated which follows directly from the $\mathcal{N}=1 \mathrm{SUSY}$ transformations. In our Alice string construction the complex adjoint scalar breaks the symmetry to $\mathrm{O}(2)$. This $\mathrm{O}(2)$ consists of a rotation around two different axis, that is, an $\mathrm{SO}(2)$ rotation around $\tau^{1}$ and a $\pi$ rotation around $\tau^{2}, \tau^{3}$ or their linear combination $\left(\mathbb{Z}_{2}\right)$. These two rotations actually do not commute with each other. This generates the ambiguity in the presence of a vortex when the generator of $\mathrm{SO}(2)$ becomes space dependent; once it encircles the Alice string, it rotates around $\tau^{2,3}$ by $\pi$ in the internal space. This implies that the element of $\mathbb{Z}_{2}$ acts non-trivially on the generator of the unbroken $\mathrm{SO}(2)$, which changes the sign.

Alice strings found in this paper are the first explicit construction of BPS Alice strings in SUSY gauge theory. It will be interesting to investigate what impact it has on various properties such as Cheshire charges in SUSY gauge theories. It will be also possible to realize our SUSY gauge theory in D-brane configurations with an orientifold in string theory. It may be related to a D-brane configuration discussed in refs. [29, 30]. It would be interesting to ask what are the consequences on D-brane physics of our BPS construction. ${ }^{3}$

Since the presence of our BPS Alice string breaks the $\mathrm{O}(2)$ symmetry around the vortex core, it produces a Nambu-Goldstone mode localized around the core giving rise to a U(1) modulus. We have constructed the low energy effective theory explicitly in ref. [44]. In the SUSY context, there will be also fermion zero modes on the Alice string that constitute a SUSY multiplet of unbroken SUSY. However, these modes will be non-normalizable as in refs. $[4,5]$, while a relative $\mathrm{U}(1)$ modulus of two Alice strings might be normalizable as in semi-local strings [45] since two strings would not exhibit Alice properties.

In this paper, we have constructed only a single vortex solution. The next step will be constructing multi-vortex configurations. Arbitrary number of Alice strings should be able to be placed at any positions since our system is BPS and there should be no force among them. We expect that odd number of Alice strings have Alice property while even number of them have no such property.

Our model admits a monopole since $\pi_{2}\left[\left(S^{1} \times S^{2}\right) / \mathbb{Z}_{2}\right]=\mathbb{Z}$. In fact, a global monopole was constructed in spin-1 BEC [46]. The interesting is that this falls into an Alice string ring with twisted $\mathrm{U}(1)$ modulus, as is the case of a monopole in the conventional Alice

\footnotetext{
${ }^{3}$ Alice strings are also discussed in the context of defect branes in string theory [43].
} 
theory $[12,14]$. It is interesting question whether our model admits a BPS monopole and whether it is also a form of a twisted Alice ring.

In this paper we have considered only the simplest model with $\mathrm{U}(1) \times \mathrm{SO}(3)$ gauge theory with charged complex scalar fields in the vector representation or equivalently $\mathrm{U}(1) \times$ $\mathrm{SU}(2)$ gauge theory with charged complex adjoint scalar fields. The simplest generalizations of our model will be charged complex scalar fields in any spin representation of $\mathrm{SO}(3)$, $\mathrm{U}(1) \times \mathrm{SO}(N)$ gauge theory with charged complex scalar fields in the vector (or other) representation, and $\mathrm{U}(1) \times \mathrm{SU}(N)$ gauge theory with charged complex scalar fields in the adjoint representation. In these cases, an unbroken non-Abelian gauge group in the bulk would have an obstruction in its continuos component (in addition to the Alice property in $\mathbb{Z}_{2}$ ), and it is broken in the vortex core giving rise to non-normalizable non-Abelian moduli as in ref. [39].

\section{Acknowledgments}

This work is supported by the Ministry of Education, Culture, Sports, Science (MEXT)Supported Program for the Strategic Research Foundation at Private Universities "Topological Science" (Grant No. S1511006). C. C. acknowledges support as an International Research Fellow of the Japan Society for the Promotion of Science (JSPS) (Grant No: 16F16322). The work of M. N. is supported in part by JSPS Grant-in-Aid for Scientific Research (KAKENHI Grant No. 25400268), and by a Grant-in-Aid for Scientific Research on Innovative Areas "Topological Materials Science" (KAKENHI Grant No. 15H05855) and "Nuclear Matter in Neutron Stars Investigated by Experiments and Astronomical Observations" (KAKENHI Grant No. 15H00841) from the Ministry of Education, Culture, Sports, Science (MEXT) of Japan.

Open Access. This article is distributed under the terms of the Creative Commons Attribution License (CC-BY 4.0), which permits any use, distribution and reproduction in any medium, provided the original author(s) and source are credited.

\section{References}

[1] A.S. Schwarz, Field theories with no local conservation of the electric charge, Nucl. Phys. B 208 (1982) 141 [INSPIRE].

[2] J.E. Kiskis, Disconnected gauge groups and the global violation of charge conservation, Phys. Rev. D 17 (1978) 3196 [INSPIRE].

[3] S.I. Blinnikov and M. Yu. Khlopov, On possible effects of 'mirror' particles, Sov. J. Nucl. Phys. 36 (1982) 472 [Yad. Fiz. 36 (1982) 809] [INSPIRE].

[4] M.G. Alford, K. Benson, S.R. Coleman, J. March-Russell and F. Wilczek, The interactions and excitations of non-Abelian vortices, Phys. Rev. Lett. 64 (1990) 1632 [Erratum ibid. 65 (1990) 668] [INSPIRE].

[5] M.G. Alford, K. Benson, S.R. Coleman, J. March-Russell and F. Wilczek, Zero modes of non-Abelian vortices, Nucl. Phys. B 349 (1991) 414 [INSPIRE].

[6] M.G. Alford, K.-M. Lee, J. March-Russell and J. Preskill, Quantum field theory of non-Abelian strings and vortices, Nucl. Phys. B 384 (1992) 251 [hep-th/9112038] [INSPIRE]. 
[7] J. Preskill and L.M. Krauss, Local discrete symmetry and quantum mechanical hair, Nucl. Phys. B 341 (1990) 50 [INSPIRE].

[8] M. Bucher and A. Goldhaber, $\mathrm{SO}(10)$ cosmic strings and $\mathrm{SU}(3)$-color Cheshire charge, Phys. Rev. D 49 (1994) 4167 [hep-ph/9310262] [INSPIRE].

[9] M. Bucher, H.-K. Lo and J. Preskill, Topological approach to Alice electrodynamics, Nucl. Phys. B 386 (1992) 3 [hep-th/9112039] [INSPIRE].

[10] H.-K. Lo and J. Preskill, Non-Abelian vortices and non-Abelian statistics, Phys. Rev. D 48 (1993) 4821 [hep-th/9306006] [INSPIRE].

[11] R. Shankar, The SO(3) monopole catalog, Phys. Rev. D 14 (1976) 1107 [InSPIRE].

[12] F.A. Bais and J. Striet, On a core instability of 't Hooft-Polyakov monopoles, Phys. Lett. B 540 (2002) 319 [hep-th/0205152] [INSPIRE].

[13] J. Striet and F.A. Bais, More on core instabilities of magnetic monopoles, JHEP 06 (2003) 022 [hep-th/0304189] [inSPIRE].

[14] K.M. Benson and T. Imbo, Topologically Alice strings and monopoles, Phys. Rev. D 70 (2004) 025005 [hep-th/0407001] [INSPIRE].

[15] E.B. Bogomolny, Stability of classical solutions, Sov. J. Nucl. Phys. 24 (1976) 449 [Yad. Fiz. 24 (1976) 861] [INSPIRE].

[16] M.K. Prasad and C.M. Sommerfield, An exact classical solution for the 't Hooft monopole and the Julia-Zee dyon, Phys. Rev. Lett. 35 (1975) 760 [InSPIRE].

[17] A.A. Abrikosov, On the magnetic properties of superconductors of the second group, Sov. Phys. JETP 5 (1957) 1174 [Zh. Eksp. Teor. Fiz. 32 (1957) 1442] [INSPIRE].

[18] H.B. Nielsen and P. Olesen, Vortex line models for dual strings, Nucl. Phys. B 61 (1973) 45 [INSPIRE].

[19] E. Witten and D.I. Olive, Supersymmetry algebras that include topological charges, Phys. Lett. B 78 (1978) 97 [INSPIRE].

[20] A. Hanany and D. Tong, Vortices, instantons and branes, JHEP 07 (2003) 037 [hep-th/0306150] [INSPIRE].

[21] R. Auzzi, S. Bolognesi, J. Evslin, K. Konishi and A. Yung, Non-Abelian superconductors: vortices and confinement in $N=2$ SQCD, Nucl. Phys. B 673 (2003) 187 [hep-th/0307287] [INSPIRE].

[22] A. Hanany and D. Tong, Vortex strings and four-dimensional gauge dynamics, JHEP 04 (2004) 066 [hep-th/0403158] [INSPIRE].

[23] M. Shifman and A. Yung, Non-Abelian string junctions as confined monopoles, Phys. Rev. D 70 (2004) 045004 [hep-th/0403149] [INSPIRE].

[24] A. Gorsky, M. Shifman and A. Yung, Non-Abelian Meissner effect in Yang-Mills theories at weak coupling, Phys. Rev. D 71 (2005) 045010 [hep-th/0412082] [InSPIRE].

[25] M. Eto, Y. Isozumi, M. Nitta, K. Ohashi and N. Sakai, Moduli space of non-Abelian vortices, Phys. Rev. Lett. 96 (2006) 161601 [hep-th/0511088] [INSPIRE].

[26] M. Eto et al., Non-Abelian vortices of higher winding numbers, Phys. Rev. D 74 (2006) 065021 [hep-th/0607070] [INSPIRE].

[27] M. Eto, K. Hashimoto, G. Marmorini, M. Nitta, K. Ohashi and W. Vinci, Universal reconnection of non-Abelian cosmic strings, Phys. Rev. Lett. 98 (2007) 091602 [hep-th/0609214] [INSPIRE]. 
[28] M. Eto, Y. Isozumi, M. Nitta, K. Ohashi and N. Sakai, Solitons in the Higgs phase: the moduli matrix approach, J. Phys. A 39 (2006) R315 [hep-th/0602170] [INSPIRE].

[29] J.A. Harvey and A.B. Royston, Localized modes at a D-brane-O-plane intersection and heterotic Alice atrings, JHEP 04 (2008) 018 [arXiv:0709.1482] [INSPIRE].

[30] J.A. Harvey and A.B. Royston, Gauge/gravity duality with a chiral $N=(0,8)$ string defect, JHEP 08 (2008) 006 [arXiv: 0804.2854] [INSPIRE].

[31] J. Striet and F.A. Bais, Simple models with Alice fluxes, Phys. Lett. B 497 (2000) 172 [hep-th/0010236] [INSPIRE].

[32] U. Leonhardt and G.E. Volovik, How to create Alice string (half quantum vortex) in a vector Bose-Einstein condensate, Pisma Zh. Eksp. Teor. Fiz. 72 (2000) 66 [JETP Lett. 72 (2000) 46] [cond-mat/0003428] [INSPIRE].

[33] S. Kobayashi, M. Kobayashi, Y. Kawaguchi, M. Nitta and M. Ueda, Abe homotopy classification of topological excitations under the topological influence of vortices, Nucl. Phys. B 856 (2012) 577 [arXiv:1110.1478] [INSPIRE].

[34] Y. Kawaguchi and M. Ueda, Spinor Bose-Einstein condensates, Phys. Rept. 520 (2012) 253 [INSPIRE].

[35] C.H. Taubes, Arbitrary N: vortex solutions to the first order Landau-Ginzburg equations, Commun. Math. Phys. 72 (1980) 277 [InSPIRE].

[36] P.C. Nelson and A. Manohar, Global color is not always defined, Phys. Rev. Lett. 50 (1983) 943 [INSPIRE].

[37] A. Abouelsaood, Are there chromodyons?, Nucl. Phys. B 226 (1983) 309 [INSPIRE].

[38] A.P. Balachandran, G. Marmo, N. Mukunda, J.S. Nilsson, E.C.G. Sudarshan and F. Zaccaria, Monopole topology and the problem of color, Phys. Rev. Lett. 50 (1983) 1553 [INSPIRE].

[39] S. Bolognesi, C. Chatterjee, J. Evslin, K. Konishi, K. Ohashi and L. Seveso, Geometry and dynamics of a coupled 4D-2D quantum field theory, JHEP 01 (2016) 075 [arXiv: 1509.04061] [INSPIRE].

[40] S.C. Davis, A.C. Davis and M. Trodden, Cosmic strings, zero modes and SUSY breaking in non-Abelian $N=1$ gauge theories, Phys. Rev. D 57 (1998) 5184 [hep-ph/9711313] [INSPIRE].

[41] A. Achucarro, A.C. Davis, M. Pickles and J. Urrestilla, Vortices in theories with flat directions, Phys. Rev. D 66 (2002) 105013 [hep-th/0109097] [INSPIRE].

[42] A.A. Penin, V.A. Rubakov, P.G. Tinyakov and S.V. Troitsky, What becomes of vortices in theories with flat directions, Phys. Lett. B 389 (1996) 13 [hep-ph/9609257] [INSPIRE].

[43] T. Okada and Y. Sakatani, Defect branes as Alice strings, JHEP 03 (2015) 131 [arXiv: 1411.1043] [INSPIRE].

[44] C. Chatterjee and M. Nitta, The effective action of a BPS Alice string, arXiv:1706.10212 [INSPIRE].

[45] M. Eto et al., On the moduli space of semilocal strings and lumps, Phys. Rev. D 76 (2007) 105002 [arXiv: 0704.2218] [INSPIRE].

[46] J. Ruostekoski and J.R. Anglin, Monopole core instability and Alice rings in spinor Bose-Einstein condensates, Phys. Rev. Lett. 91 (2003) 190402 [Erratum ibid. 97 (2006) 069902] [cond-mat/0307651] [INSPIRE]. 